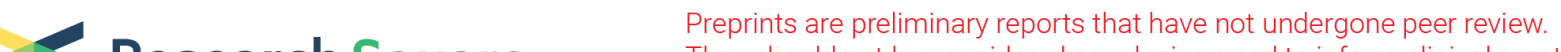 $\begin{array}{ll}\text { Research Square } & \text { They should not be considered conclusive, used to inform clinical practice, } \\ \text { or referenced by the media as validated information. }\end{array}$
}

\section{An Operator-theoretical Study on the BCS- Bogoliubov Model of Superconductivity Near Absolute Zero Temperature}

Shuji Watanabe ( $\nabla$ shuwatanabe@gunma-u.ac.jp)

Division of Mathematical Sciences Graduate School of Engineering, Gunma University 4-2 Aramakimachi, Maebashi 371-8510, Japan

\section{Research Article}

Keywords: Entropy, specific heat at constant volume, critical magnetic field, BCS-Bogoliubov gap equation, BCS-Bogoliubov model, superconductivity

Posted Date: January 15th, 2021

DOI: https://doi.org/10.21203/rs.3.rs-145490/v1

License: (a) (i) This work is licensed under a Creative Commons Attribution 4.0 International License. Read Full License

Version of Record: A version of this preprint was published at Scientific Reports on August 5th, 2021. See the published version at https://doi.org/10.1038/s41598-021-95322-x. 


\title{
An operator-theoretical study on the BCS-Bogoliubov model of superconductivity near absolute zero temperature
}

\author{
Shuji Watanabe \\ Division of Mathematical Sciences \\ Graduate School of Engineering, Gunma University \\ 4-2 Aramaki-machi, Maebashi 371-8510, Japan \\ Email: shuwatanabe@gunma-u.ac.jp
}

\begin{abstract}
In the BCS-Bogoliubov model of superconductivity, no one gave a proof of the statement that the solution to the BCS-Bogoliubov gap equation is differentiable with respect to the temperature. But, without such a proof, one differentiates the solution and the thermodynamic potential with respect to the temperature twice, and one obtains the entropy and the specific heat at constant volume of a superconductor. In the preceding papers, the present author showed that the solution is indeed differentiable with respect to the temperature twice. Thanks to these results, we in this paper differentiate the thermodynamic potential with respect to the temperature twice so as to obtain the entropy and the specific heat at constant volume from the viewpoint of operator theory. Here, the potential in the BCS-Bogoliubov gap equation is a function and need not be a constant. We then show the behavior near absolute zero temperature of the entropy, the specific heat, the solution and the critical magnetic field.
\end{abstract}

Mathematics Subject Classification 2020. 45G10, 47H10, 47N50, 82D55.

Keywords. Entropy, specific heat at constant volume, critical magnetic field, BCS-Bogoliubov gap equation, BCS-Bogoliubov model, superconductivity.

\section{Introduction}

In the BCS-Bogoliubov model of superconductivity, no one gave a proof of the statement that the solution to the BCS-Bogoliubov gap equation is partially differentiable with respect to the temperature. But, without such a proof, one partially differentiates the solution and the thermodynamic potential with respect to the temperature twice, and one shows that the phase transition to a superconducting state is of the second order. So, if the solution were not partially differentiable with respect to the temperature, then one could not show that the phase transition is of the second order. Moreover, one could not obtain the entropy and the specific heat at constant volume differentiating the solution and the thermodynamic potential with respect to the temperature. Therefore, we have to show that the solution is partially differentiable with respect to the temperature twice.

On the basis of fixed-point theorems, the present author [1, Theorems 2.3 and 2.4] (see also [2, Theorems 2.2 and 2.10]) showed that the solution is indeed partially differentiable with respect to the temperature twice, and showed that the thermodynamic potential is also differentiable with respect to the temperature twice. Here, the potential in the BCS-Bogoliubov gap equation 
is a function and need not be a constant. In this way, the present author gave an operatortheoretical proof of the statement that the phase transition from a normal conducting state to a superconducting state is of the second order, and solved the long-standing problem of the second-order phase transition from the viewpoint of operator theory. As s result, the present author showed the existence of the first and second order partial derivatives of the solution with respect to the temperature as well as the existence and uniqueness of the solution itself. Moreover, the present author showed that all of the solution, the first and second order partial derivatives are continuous functions of both the temperature and the energy. Therefore, thanks to these results, we can indeed differentiate the thermodynamic potential with respect to the temperature twice so as to obtain the entropy and the specific heat at constant volume.

Let $u_{0}$ be the solution to the BCS-Bogoliubov gap equation $[3,4]$, which is a nonlinear integral equation and is given by

$$
u_{0}(T, x)=\int_{\varepsilon}^{\hbar \omega_{D}} \frac{U(x, \xi) u_{0}(T, \xi)}{\sqrt{\xi^{2}+u_{0}(T, \xi)^{2}}} \tanh \frac{\sqrt{\xi^{2}+u_{0}(T, \xi)^{2}}}{2 T} d \xi, T \geq 0, x, \xi \in\left[\varepsilon, \hbar \omega_{D}\right]
$$

Here, the solution $u_{0}$ is a function of the absolute temperature $T$ and the energy $x$. The Debye angular frequency $\omega_{D}$ is a positive constant and depends on a superconductor. The potential $U(\cdot, \cdot)$ satisfies $U(x, \xi)>0$ at all $(x, \xi) \in\left[\varepsilon, \hbar \omega_{D}\right]^{2}$. Throughout this paper we use the unit where the Boltzmann constant $k_{B}$ is equal to 1 .

Remark 1.1. In (1.1) above and (1.2) below, we introduce a cutoff $\varepsilon>0$ and fix it. If we did not introduce the cutoff $\varepsilon>0$, then the first order derivative of the thermodynamic potential with respect to $T$, and hence the entropy could diverge logarithmically only at the transition temperature. Therefore, the phase transition could not be of the second order. This contradicts a lot of experimental results that the phase transition is of the second order without an external magnetic field. So we introduce the cutoff $\varepsilon>0$. For more details, see [2, Remarks 1.1, 1.10 and 1.11].

For a fixed temperature $T$, the existence and uniqueness of the solution were established and studied in $[5,6,7,8,9,10,11,12,13,14,15,16,17,18,19,20,21,1,2]$. See also Kuzemsky [22, Chapters 26 and 29] and [23, 24]. For the role of the chemical potential in the BCS-Bogoliubov model, see Anghel and Nemnes [25] and Anghel [26, 27].

In conection to this, the BCS-Bogoliubov gap equation plays a role similar to that of the Maskawa-Nakajima equation [28, 29] which has attracted considerable interest in elementary particle physics. In Professor Maskawa's Nobel lecture, he stated the reason why he dealt with the Maskawa-Nakajima equation. For an operator-theoretical treatment of this equation, see the present author's paper [30].

In the BCS-Bogoliubov model, the thermodynamic potential is given by

$$
\begin{aligned}
\Omega(T)= & -2 N_{0} \int_{\varepsilon}^{\hbar \omega_{D}} \sqrt{x^{2}+u_{0}(T, x)^{2}} d x \\
& +N_{0} \int_{\varepsilon}^{\hbar \omega_{D}} \frac{u_{0}(T, x)^{2}}{\sqrt{x^{2}+u_{0}(T, x)^{2}}} \tanh \frac{\sqrt{x^{2}+u_{0}(T, x)^{2}}}{2 T} d x \\
& -4 N_{0} T \int_{\varepsilon}^{\hbar \omega_{D}} \ln \left(1+e^{-\sqrt{x^{2}+u_{0}(T, x)^{2}} / T}\right) d x, \quad 0 \leq T \leq T_{c}
\end{aligned}
$$

where $u_{0}$ is the solution to the BCS-Bogoliubov gap equation (1.1), $T_{c}$ is the transition temperature (see [2, Definition 1.8] for our operator-theoretical definition of $T_{c}$ ) and $N_{0}$ is a positive constant and denotes the density of states per unit energy at the Fermi surface. Here we consider 
only the contribution from the interval $\left[-\hbar \omega_{D}, \hbar \omega_{D}\right]$, and omit the contribution from the other intervals. In other words, we consider only the contribution from superconductivity. For more details, see $[2,(1.5)$ and (1.6)].

As mentioned above, thanks to [1, Theorems 2.3 and 2.4] and [2, Theorems 2.2 and 2.10]), we can indeed partially differentiate the solution with respect to the temperature $T$ twice, and have the solution $u_{0}$, the first order partial derivative $\left(\partial u_{0} / \partial T\right)$ and the second order partial derivative $\left(\partial^{2} u_{0} / \partial T^{2}\right)$. Moreover, all of them are continuous functions of both the temperature $T$ and the energy $x$. Therefore, thanks to these results, we can indeed differentiate the thermodynamic potential $\Omega$ with respect to $T$ twice so as to obtain the entropy and the specific heat at constant volume. Note that the potential $U(\cdot, \cdot)$ in the BCS-Bogoliubov gap equation is a function and need not be a constant.

Remark 1.2. If the solution $u_{0}$ is an accumulating point of the set $V$ in [2, Theorem 2.2] (resp. of the set $W$ in [2, Theorem 2.10]), then we replace $u_{0}$ by a suitably chosen element of $V$ (resp. of $W$ ) in the form (1.2) of the thermodynamic potential $\Omega$. This is because $u_{0}$ is an accumulating point. Note that such a suitably chosen element is partially differentiable with respect to the temperature $T$ twice and that it is a continuous function of both the temperature $T$ and the energy $x$. Therefore, once we replace the solution $u_{0}$ by a suitably chosen element in the form (1.2) of the thermodynamic potential $\Omega$, we can differentiate the thermodynamic potential $\Omega$ with respect to the temperature $T$ twice so as to obtain the entropy and the specific heat at constant volume.

\section{Main results}

Thanks to Theorem 2.2 in [2], the solution $u_{0}$ to the BCS-Bogoliubov gap equation (1.1) satisfies that at all $x \in\left[\varepsilon, \hbar \omega_{D}\right]$,

$$
\frac{\partial u_{0}}{\partial T}(0, x)=0 \quad \text { and } \quad \frac{\partial^{2} u_{0}}{\partial T^{2}}(0, x)=0 .
$$

Note that all of the solution $u_{0}$, the first order partial derivative $\left(\partial u_{0} / \partial T\right)$ and the second order partial derivative $\left(\partial^{2} u_{0} / \partial T^{2}\right)$ are continuous functions of both the temperature $T$ and the energy $x$. Therefore, throughout the paper, we apply the following approximation to the solution $u_{0}$, the first order partial derivative $\left(\partial u_{0} / \partial T\right)$ and the second order partial derivative $\left(\partial^{2} u_{0} / \partial T^{2}\right)$ :

Approximation (A) If $T \simeq 0$, then

$\frac{\partial u_{0}}{\partial T}(T, x) \simeq \frac{\partial u_{0}}{\partial T}(0, x)(=0), \frac{\partial^{2} u_{0}}{\partial T^{2}}(T, x) \simeq \frac{\partial^{2} u_{0}}{\partial T^{2}}(0, x)(=0)$, and hence $u_{0}(T, x) \simeq u_{0}(0, x)$

at all $x \in\left[\varepsilon, \hbar \omega_{D}\right]$. Moreover, if $T \simeq 0$, then

$$
\frac{(X / T)^{n}}{\cosh (X / T)} \simeq 0 \quad(X>0)
$$

for every nonnegative integer $n$.

Remark 2.1. The approximation $u_{0}(T, x) \simeq u_{0}(0, x)$ above follows from $\left(\partial u_{0} / \partial T\right)(0, x)=0$ and $\left(\partial^{2} u_{0} / \partial T^{2}\right)(T, x) \simeq 0$.

Theorem 2.2. Let $u_{0}$ be the solution to the BCS-Bogoliubov gap equation (1.1) given by Theorem 2.2 in [2]. Suppose Approximation (A). Then the thermodynamic potential $\Omega$ is partially 
differentiable with respect to the temperature $T$ twice, and so there exist the entropy $S$ and the specific heat $C_{V}$ at constant volume. The entropy $S$, the specific heat $C_{V}$ at constant volume and the solution $u_{0}$ behave at $T \simeq 0$ as follows:

$$
\begin{aligned}
S(T) & \simeq \frac{4 N_{0}}{T} \int_{\varepsilon}^{\hbar \omega_{D}} \sqrt{\xi^{2}+u_{0}(0, \xi)^{2}} \exp \left(-\frac{\sqrt{\xi^{2}+u_{0}(0, \xi)^{2}}}{T}\right) d \xi \\
C_{V}(T) & \simeq \frac{4 N_{0}}{T^{2}} \int_{\varepsilon}^{\hbar \omega_{D}}\left\{\xi^{2}+u_{0}(0, \xi)^{2}\right\} \exp \left(-\frac{\sqrt{\xi^{2}+u_{0}(0, \xi)^{2}}}{T}\right) d \xi \\
u_{0}(T, x) & \simeq u_{0}(0, x)-2 \int_{\varepsilon}^{\hbar \omega_{D}} U(x, \xi) \exp \left(-\frac{\sqrt{\xi^{2}+u_{0}(0, \xi)^{2}}}{T}\right) d \xi .
\end{aligned}
$$

Moreover, the critical magnetic field at absolute zero temperature and the specific heat at the transition temperature $T_{c}$ satisfy

$$
\frac{H_{c}(0)^{2}}{T_{c} C_{V}\left(T_{c}\right)}=\frac{4 \pi}{\int_{\varepsilon /\left(2 T_{c}\right)}^{\hbar \omega_{D} /\left(2 T_{c}\right)} \frac{\eta^{2}}{\cosh ^{2} \eta} d \eta} \int_{\varepsilon /\left(2 T_{c}\right)}^{\hbar \omega_{D} /\left(2 T_{c}\right)} \frac{\left\{\sqrt{\eta^{2}+\left(2 T_{c}\right)^{-2} u_{0}\left(0,2 T_{c} \eta\right)^{2}}-\eta\right\}^{2}}{\sqrt{\eta^{2}+\left(2 T_{c}\right)^{-2} u_{0}\left(0,2 T_{c} \eta\right)^{2}}} d \eta .
$$

Remark 2.3. Since $\hbar \omega_{D} /\left(2 T_{c}\right)$ is very large in many superconductors, we often let $\hbar \omega_{D} /\left(2 T_{c}\right) \rightarrow$ $\infty$ and $\varepsilon /\left(2 T_{c}\right) \rightarrow 0$ in the physics literature.

Corollary 2.4. Suppose that $u_{0}\left(0,2 T_{c} \eta\right) / T_{c}$ is a constant and does not depend on superconductors, and let $\hbar \omega_{D} /\left(2 T_{c}\right) \rightarrow \infty$ and $\varepsilon /\left(2 T_{c}\right) \rightarrow 0$. Then $H_{c}(0)^{2} /\left(T_{c} C_{V}\left(T_{c}\right)\right)$ does not depend on superconductors and becomes a universal constant.

Remark 2.5. As far as the present author knows, similar results are obtained in the physics literature under the restriction that the potential $U(\cdot, \cdot)$ in the BCS-Bogoliubov gap equation is a constant. But Theorem 2.2 holds true even when the potential $U(\cdot, \cdot)$ is not a constant but a function.

Remark 2.6. Suppose that the potential $U(\cdot, \cdot)$ is a constant, i.e., $U(\cdot, \cdot)=U_{0}$. Here, $U_{0}$ is a positive constant. Then the solution $u_{0}$ to the BCS-Bogoliubov gap equation does not depend on the energy $x$ and becomes a function of the temperature $T$ only. We denote the solution by $u_{0}(T)$. Then the forms of $S(T), C_{V}(T)$ and $u_{0}(T, x)$ in Theorem 2.2 are reduced to the following well-known forms, respectively: At $T \simeq 0$,

$$
\begin{aligned}
& S(T) \simeq \frac{2 \sqrt{2 \pi} N_{0} u_{0}(0)^{3 / 2}}{\sqrt{T}} \exp \left(-\frac{u_{0}(0)}{T}\right), \quad C_{V}(T) \simeq \frac{2 \sqrt{2 \pi} N_{0} u_{0}(0)^{5 / 2}}{T^{3 / 2}} \exp \left(-\frac{u_{0}(0)}{T}\right), \\
& u_{0}(T) \simeq u_{0}(0)-U_{0} \sqrt{2 \pi T u_{0}(0)} \exp \left(-\frac{u_{0}(0)}{T}\right)
\end{aligned}
$$

as $\hbar \omega_{D} /\left(2 T_{c}\right) \rightarrow \infty$ and $\varepsilon /\left(2 T_{c}\right) \rightarrow 0$.

Remark 2.7. Suppose that the potential $U(\cdot, \cdot)$ is a constant. Then the solution $u_{0}$ becomes a function of the temperature $T$ only, and the form of $H_{c}(0)^{2} /\left(T_{c} C_{V}\left(T_{c}\right)\right)$ in Theorem 2.2 is reduced to

$$
\frac{H_{c}(0)^{2}}{T_{c} C_{V}\left(T_{c}\right)}=\frac{4 \pi}{\int_{\varepsilon /\left(2 T_{c}\right)}^{\hbar \omega_{D} /\left(2 T_{c}\right)} \frac{\eta^{2}}{\cosh ^{2} \eta} d \eta} \int_{\varepsilon /\left(2 T_{c}\right)}^{\hbar \omega_{D} /\left(2 T_{c}\right)} \frac{\left\{\sqrt{\eta^{2}+\left(2 T_{c}\right)^{-2} u_{0}(0)^{2}}-\eta\right\}^{2}}{\sqrt{\eta^{2}+\left(2 T_{c}\right)^{-2} u_{0}(0)^{2}}} d \eta .
$$


Therefore, if $u_{0}(0) / T_{c}$ does not depend on superconductors, then $H_{c}(0)^{2} /\left(T_{c} C_{V}\left(T_{c}\right)\right)$ does not depend on superconductors and becomes a universal constant as $\hbar \omega_{D} /\left(2 T_{c}\right) \rightarrow \infty$ and $\varepsilon /\left(2 T_{c}\right) \rightarrow$ 0 . Actually, $u_{0}(0) / T_{c}$ does not depend on superconductors since

$$
u_{0}(0) / T_{c} \simeq 4 \exp \left[\int_{0}^{\infty}(\ln \eta) /\left(\cosh ^{2} \eta\right) d \eta\right]
$$

as is shown in the physics literature.

\section{Proof of Theorem 2.2}

We first give a proof for the behavior of the entropy $S$ at $T \simeq 0$ in Theorem 2.2. Thanks to [1, Theorems 2.3 and 2.4] and [2, Theorems 2.2 and 2.10], we can indeed partially differentiate the solution $u_{0}$ to the BCS-Bogoliubov gap equation with respect to $T$ twice. Therefore, we can also differentiate the thermodynamic potential $\Omega$ with respect to $T$ twice. A straightforward calculation gives

$$
\begin{aligned}
& \frac{\partial \Omega}{\partial T}(T)=-N_{0} \int_{\varepsilon}^{\hbar \omega_{D}} \frac{1}{\sqrt{\xi^{2}+u_{0}(T, \xi)^{2}}} \frac{\partial u^{2}}{\partial T}(T, \xi) d \xi \\
& +N_{0} \int_{\varepsilon}^{\hbar \omega_{D}} \frac{\partial u^{2}}{\partial T}(T, \xi) \frac{1}{\sqrt{\xi^{2}+u_{0}(T, \xi)^{2}}} \tanh \frac{\sqrt{\xi^{2}+u_{0}(T, \xi)^{2}}}{2 T} d \xi \\
& -\frac{N_{0}}{2} \int_{\varepsilon}^{\hbar \omega_{D}} \frac{\partial u^{2}}{\partial T}(T, \xi) \frac{u_{0}(T, \xi)^{2}}{\left(\xi^{2}+u_{0}(T, \xi)^{2}\right)^{3 / 2}} \tanh \frac{\sqrt{\xi^{2}+u_{0}(T, \xi)^{2}}}{2 T} d \xi \\
& +\frac{N_{0}}{4 T} \int_{\varepsilon}^{\hbar \omega_{D}} \frac{\partial u^{2}}{\partial T}(T, \xi) \frac{u_{0}(T, \xi)^{2}}{\xi^{2}+u_{0}(T, \xi)^{2}}\left(\cosh \frac{\sqrt{\xi^{2}+u_{0}(T, \xi)^{2}}}{2 T}\right)^{-2} d \xi \\
& -\frac{N_{0}}{2 T^{2}} \int_{\varepsilon}^{\hbar \omega_{D}} u_{0}(T, \xi)^{2}\left(\cosh \frac{\sqrt{\xi^{2}+u_{0}(T, \xi)^{2}}}{2 T}\right)^{-2} \\
& -4 N_{0} \int_{\varepsilon}^{\hbar \omega_{D}} \ln \left(1+e^{-\sqrt{\xi^{2}+u_{0}(T, \xi)^{2}} / T}\right) d \xi \\
& -4 N_{0} \int_{\varepsilon}^{\hbar \omega_{D}} \frac{1}{e^{\sqrt{\xi^{2}+u_{0}(T, \xi)^{2}} / T}+1}\left\{\frac{\sqrt{\xi^{2}+u_{0}(T, \xi)^{2}}}{T}\right. \\
& \left.-\frac{\frac{\partial u^{2}}{\partial T}(T, \xi)}{2 \sqrt{\xi^{2}+u_{0}(T, \xi)^{2}}}\right\} d \xi
\end{aligned}
$$

where $0 \leq T \leq T_{c}$.

Under Approximation (A), we thus have the behavior for the entropy $S$ at $T \simeq 0$ :

$$
\begin{aligned}
S(T) & =-\frac{\partial \Omega}{\partial T}(T) \simeq \frac{4 N_{0}}{T} \int_{\varepsilon}^{\hbar \omega_{D}} \frac{\sqrt{\xi^{2}+u_{0}(0, \xi)^{2}}}{e^{\sqrt{\xi^{2}+u_{0}(0, \xi)^{2}} / T}+1} d \xi \\
& \simeq \frac{4 N_{0}}{T} \int_{\varepsilon}^{\hbar \omega_{D}} \sqrt{\xi^{2}+u_{0}(0, \xi)^{2}} e^{-\sqrt{\xi^{2}+u_{0}(0, \xi)^{2}} / T} d \xi .
\end{aligned}
$$

Note that the sixth term on the right side of (3.1) is negligible. This is because the sixth term becomes $($ at $T \simeq 0)$

$$
-4 N_{0} \int_{\varepsilon}^{\hbar \omega_{D}} \ln \left(1+e^{-\sqrt{\xi^{2}+u_{0}(T, \xi)^{2}} / T}\right) d \xi \simeq-4 N_{0} \int_{\varepsilon}^{\hbar \omega_{D}} e^{-\sqrt{\xi^{2}+u_{0}(0, \xi)^{2}} / T} d \xi
$$


which is negligible compared to the seventh term.

We next give a proof for the behavior for the specific heat $C_{V}$ at constant volume at $T \simeq 0$. To this end we differentiate $(\partial \Omega / \partial T)$ with respect to $T$ again and obtain the second order partial derivative $\left(\partial^{2} \Omega / \partial T^{2}\right)$. The second order partial derivative of the first term on the right side of (3.1) becomes

$$
-N_{0} \int_{\varepsilon}^{\hbar \omega_{D}}\left[\frac{1}{\sqrt{\xi^{2}+u_{0}(T, \xi)^{2}}} \frac{\partial^{2} u^{2}}{\partial T^{2}}(T, \xi)-\frac{1}{2\left\{\xi^{2}+u_{0}(T, \xi)^{2}\right\}^{3 / 2}}\left\{\frac{\partial u^{2}}{\partial T}(T, \xi)\right\}^{2}\right] d \xi
$$

which turns out to be 0 at $T \simeq 0$ under Approximation (A). On the other hand, the second order partial derivative of the last term on the right side of (3.1) includes

$$
-\frac{4 N_{0}}{T^{3}} \int_{\varepsilon}^{\hbar \omega_{D}}\left\{\xi^{2}+u_{0}(T, \xi)^{2}\right\} \frac{e^{\sqrt{\xi^{2}+u_{0}(T, \xi)^{2}} / T}}{\left(e^{\sqrt{\xi^{2}+u_{0}(T, \xi)^{2}} / T}+1\right)^{2}} d \xi
$$

which is the only term that is not equal to 0 at $T \simeq 0$ under Approximation (A). We deal with the other terms on the right side of (3.1) similarly.

As a result, we obtain under Approximation $(\mathrm{A})$ that $($ at $T \simeq 0)$

$$
\frac{\partial^{2} \Omega}{\partial T^{2}}(T) \simeq-\frac{4 N_{0}}{T^{3}} \int_{\varepsilon}^{\hbar \omega_{D}}\left\{\xi^{2}+u_{0}(0, \xi)^{2}\right\} e^{-\sqrt{\xi^{2}+u_{0}(0, \xi)^{2}} / T} d \xi
$$

Therefore, under Approximation (A), we have the following behavior for the specific heat $C_{V}$ at constant volume at $T \simeq 0$ :

$$
\begin{aligned}
C_{V}(T) & =-T \frac{\partial^{2} \Omega}{\partial T^{2}}(T) \\
& \simeq \frac{4 N_{0}}{T^{2}} \int_{\varepsilon}^{\hbar \omega_{D}}\left\{\xi^{2}+u_{0}(0, \xi)^{2}\right\} e^{-\sqrt{\xi^{2}+u_{0}(0, \xi)^{2}} / T} d \xi
\end{aligned}
$$

We give a proof for the behavior for the solution $u_{0}$ at $T \simeq 0$. A straightforward calculation gives

$$
\begin{aligned}
u_{0}(T, x)-u_{0}(0, x)= & \int_{\varepsilon}^{\hbar \omega_{D}} U(x, \xi)\left\{\frac{u_{0}(T, \xi)}{\sqrt{\xi^{2}+u_{0}(T, \xi)^{2}}}-\frac{u_{0}(0, \xi)}{\sqrt{\xi^{2}+u_{0}(0, \xi)^{2}}}\right\} d \xi \\
& -2 \int_{\varepsilon}^{\hbar \omega_{D}} U(x, \xi) \frac{1}{e^{\frac{\sqrt{\xi^{2}+u_{0}(T, \xi)^{2}}}{T}}+1} d \xi .
\end{aligned}
$$

Approximation (A) implies

$$
\frac{u_{0}(T, \xi)}{\sqrt{\xi^{2}+u_{0}(T, \xi)^{2}}} \simeq \frac{u_{0}(0, \xi)}{\sqrt{\xi^{2}+u_{0}(0, \xi)^{2}}} .
$$

Therefore, we have the behavior for the solution $u_{0}$ at $T \simeq 0$ :

$$
u_{0}(T, x) \simeq u_{0}(0, x)-2 \int_{\varepsilon}^{\hbar \omega_{D}} U(x, \xi) e^{-\frac{\sqrt{\xi^{2}+u_{0}(0, \xi)^{2}}}{T}} d \xi .
$$


We finally give a proof for the rest of Theorem 2.2. Note that Theorem 2.19 (v) in [2] gives

$$
H_{c}(0)^{2}=32 \pi N_{0} T_{c}^{2} \int_{\varepsilon /\left(2 T_{c}\right)}^{\hbar \omega_{D} /\left(2 T_{c}\right)} \frac{\left\{\sqrt{\eta^{2}+\left(2 T_{c}\right)^{-2} u_{0}\left(0,2 T_{c} \eta\right)^{2}}-\eta\right\}^{2}}{\sqrt{\eta^{2}+\left(2 T_{c}\right)^{-2} u_{0}\left(0,2 T_{c} \eta\right)^{2}}} d \eta .
$$

Moreover, Lemma 5.2 in [2] gives

$$
C_{V}^{N}\left(T_{c}\right)=8 T_{c} \int_{\varepsilon /\left(2 T_{c}\right)}^{\hbar \omega_{D} /\left(2 T_{c}\right)} \frac{N_{0} \eta^{2}}{\cosh ^{2} \eta} d \eta
$$

Therefore,

$$
\frac{H_{c}(0)^{2}}{T_{c} C_{V}^{N}\left(T_{c}\right)}=\frac{4 \pi}{\int_{\varepsilon /\left(2 T_{c}\right)}^{\hbar \omega_{D} /\left(2 T_{c}\right)} \frac{\eta^{2}}{\cosh ^{2} \eta} d \eta} \int_{\varepsilon /\left(2 T_{c}\right)}^{\hbar \omega_{D} /\left(2 T_{c}\right)} \frac{\left\{\sqrt{\eta^{2}+\left(2 T_{c}\right)^{-2} u_{0}\left(0,2 T_{c} \eta\right)^{2}}-\eta\right\}^{2}}{\sqrt{\eta^{2}+\left(2 T_{c}\right)^{-2} u_{0}\left(0,2 T_{c} \eta\right)^{2}}} d \eta
$$

The proof of Theorem 2.2 is complete.

Corollary 2.4 follows immediately from the form $H_{c}(0)^{2} /\left(T_{c} C_{V}^{N}\left(T_{c}\right)\right)$ just above.

\section{References}

[1] Watanabe, S. An operator-theoretical proof for the second-order phase transition in the BCS-Bogoliubov model of superconductivity. Kyushu J. Math. 74, 177-196 (2020).

[2] Watanabe, S. An operator-theoretical study of the specific heat and the critical magnetic field in the BCS-Bogoliubov model of superconductivity. Scientific Reports 10, 9877 (2020).

[3] Bardeen, J., Cooper, L. N. \& Schrieffer, J. R. Theory of superconductivity. Phys. Rev. 108, 1175-1204 (1957).

[4] Bogoliubov, N. N. A new method in the theory of superconductivity I. Soviet Phys. JETP 34, 41-46 (1958).

[5] Odeh, F. An existence theorem for the BCS integral equation. IBM J. Res. Develop. 8, 187-188 (1964).

[6] Billard, P. \& Fano, G. An existence proof for the gap equation in the superconductivity theory. Commun. Math. Phys. 10, 274-279 (1968).

[7] Vansevenant, A. The gap equation in the superconductivity theory. Physica 17D, 339-344 (1985).

[8] Bach, V., Lieb, E. H. \& Solovej, J. P. Generalized Hartree-Fock theory and the Hubbard model. J. Stat. Phys. 76, 3-89 (1994).

[9] Chen, T., Fröhlich, J. \& Seifert, M. Renormalization Group Methods: Landau-Fermi Liquid and BCS Superconductor. Proc. of the 1994 Les Houches Summer School. arXiv:condmat/9508063.

[10] Deuchert, A., Geisinger, A., Hainzl, C. \& Loss, M. Persistence of translational symmetry in the BCS model with radial pair interaction. Ann. Henri. Poincaré 19, 1507-1527 (2018). 
[11] Frank, R. L., Hainzl, C., Naboko, S. \& Seiringer, R. The critical temperature for the BCS equation at weak coupling. J. Geom. Anal. 17, 559-568 (2007).

[12] Frank, R. L., Hainzl, C., Seiringer, R. \& Solovej, J. P. The external field dependence of the BCS critical temperature. Commun. Math. Phys. 342, 189-216 (2016).

[13] Freiji, A., Hainzl, C. \& Seiringer, R. The gap equation for spin-polarized fermions. J. Math. Phys. 53, 012101 (2012).

[14] Hainzl, C., Hamza, E., Seiringer, R. \& Solovej, J. P. The BCS functional for general pair interactions. Commun. Math. Phys. 281, 349-367 (2008).

[15] Hainzl, C. \& Loss, M. General pairing mechanisms in the BCS-theory of superconductivity. Eur. Phys. J. B, 90:82 (2017).

[16] Hainzl, C. \& Seiringer, R. Critical temperature and energy gap for the BCS equation. Phys. Rev. B 77, 184517 (2008).

[17] Hainzl, C. \& Seiringer, R. The BCS critical temperature for potentials with negative scattering length. Lett. Math. Phys. 84, 99-107 (2008).

[18] Hainzl, C. \& Seiringer, R. The Bardeen-Cooper-Schrieffer functional of superconductivity and its mathematical properties. J. Math. Phys. 57, 021101 (2016).

[19] Watanabe, S. The solution to the BCS gap equation and the second-order phase transition in superconductivity. J. Math. Anal. Appl. 383, 353-364 (2011).

[20] Watanabe, S. Addendum to 'The solution to the BCS gap equation and the second-order phase transition in superconductivity'. J. Math. Anal. Appl. 405, 742-745 (2013).

[21] Watanabe, S. \& Kuriyama, K. Smoothness and monotone decreasingness of the solution to the BCS-Bogoliubov gap equation for superconductivity. J. Basic and Applied Sciences 13, 17-25 (2017).

[22] Kuzemsky, A. L. Statistical Mechanics and the Physics of Many-Particle Model Systems. (World Scientific Publishing Co, 2017).

[23] Kuzemsky, A. L. Bogoliubov's vision: quasiaverages and broken symmetry to quantum protectorate and emergence. Internat. J. Mod. Phys. B 24, 835-935 (2010).

[24] Kuzemsky, A. L. Variational principle of Bogoliubov and generalized mean fields in manyparticle interacting systems. Internat. J. Mod. Phys. B 29, 1530010 (63 pages) (2015).

[25] Anghel, D.-V., \& Nemnes, G. A. The role of the chemical potential in the BCS theory. Physica A 464, 74-82 (2016).

[26] Anghel, D.-V. New phenomenology from an old theory-The BCS theory of superconductivity revisited. Physica A 531, 121804 (2019).

[27] Anghel, D.-V. Multiple solutions for the equilibrium populations in BCS superconductors. arXiv:1908.06017v1.

[28] Maskawa, T. \& Nakajima, H. Spontaneous breaking of chiral symmetry in a vector-gluon model. Prog. Theor. Phys. 52, 1326-1354 (1974). 
[29] Maskawa, T. \& Nakajima, H. Spontaneous breaking of chiral symmetry in a vector-gluon model II. Prog. Theor. Phys. 54, 860-877 (1975).

[30] Watanabe, S. An operator-theoretical treatment of the Maskawa-Nakajima equation in the massless abelian gluon model. J. Math. Anal. Appl. 418, 874-883 (2014).

\section{Contributions}

Shuji Watanabe wrote the main manuscript text and reviewed the manuscript.

\section{Competing interests}

The author declares no competing interests. 